\title{
Modified Bunnell suture expands the surgical indication of the treatment of Haglund's syndrome heel pain with endoscope
}

\author{
JI HUA XU, SHI-LI DING, BO CHEN and SHOU-CHENG WU \\ Department of Hand Surgery, The First Affiliated Hospital of Zhejiang University, \\ Hangzhou, Zhejiang 310002, P.R. China
}

Received September 19, 2017; Accepted November 17, 2017

DOI: $10.3892 /$ etm.2018.6071

\begin{abstract}
The aim of the present study was to develop a method which can solve the problem of partial tearing of the Achilles tendon insertion caused by the debridement for Haglund's syndrome using endoscopy-assisted percutaneous repair. Seven patients with Haglund's syndrome were prospectively recruited. All 7 patients ( 3 female, 4 male) had intratendinous calcifications. Preoperative diagnosis was made according to the clinical symptoms and diagnosis, medical examination results, plain film radiographs, and magnetic resonance imaging. The patients whose average age was 35.2 years, had experienced symptoms and were treated by conservative methods for 12-24 months (average 17.1 months). All 7 cases were treated with debridement of Achilles tendon insertion site with a standard $4.0 \mathrm{~mm}$ bur and underwent repair treatment with a modified Bunnell suture method under direct visualization using arthroscopy. The American Orthopaedic Foot and Ankle Society (AOFAS) score and the changes of the patient's parallel pitch lines were used to evaluate and assess the results. The follow-up period averaged 22 months. The lateral X-ray film after operation of all the heels of the patients showed that sufficient osseous planning of all the patients was completed. None of the patients converted to conventional open surgery. The average AOFAS scores of the 7 cases were improved significantly at final follow-up compared to pretherapy $(\mathrm{P}<0.005)$. The results of 5 of the 7 cases were excellent, 2, were good, and 0 was fair or poor. None of the cases had permanent nerve injuries, wound infections or Achilles tendon avulsion. Our study is a supplement of endoscopic repairing and strengthening of the Achilles tendon. The advantages and clinical significance of endoscopy during the treatment of Haglund's syndrome under the premise of strict control of operation indications were further verified.
\end{abstract}

Correspondence to: $\mathrm{Dr}$ Bo Chen, Department of Hand Surgery, The First Affiliated Hospital of Zhejiang University, 79 Qingchun Road, Hangzhou, Zhejiang 310002, P.R. China E-mail: chenbo3690@163.com

Key words: endoscopic, Bunnell, Achille's tendon, Haglund's syndrome

\section{Introduction}

Haglund's syndrome is recognized to cause heel pain $(1,2)$. Repeated pressure caused by calcaneus in dorsiflexion on the retrocalcaneal bursa and Achilles tendon occasionally leads to aching bursitis and insertional Achilles tendinopathy (3).

A typical patient with symptomatic Haglund's syndrome may have an aching, red, inflamed heel with an obvious osseous prominence on the lateral radiographs of the posterosuperior heel. This is always associated with insertional Achilles tendinopathy, retrocalcaneal bursitis, and superficial adventitious Achilles tendon bursitis (4). The patient complains of enlargement of the posterior heel and encounters problems with footwear and sport. The posterolateral surface becomes extremely enlarged and reddened (5).

Painful bone prominence in the retrocalcaneal region of the posterior calcaneus with no relief after 6 months of conservative therapy is considered to require surgery (6). Surgical treatment mainly includes the elimination of retrocalcaneal bursitis and visible osseous prominence, debridement and reattachment of Achilles insertion point.

Open surgical management and minimal invasive surgery are both used in the treatment of Haglund's syndrome. The advantage of the former technique is that it is a more rapid procedure, although it requires extensive exposure and it is difficult to determine the exact amount of bone resected on the medial side unless a double approach with an additional medial incision is used (5). With open surgery, a large wound in a poor blood supply area takes time to heal and various other complications have been reported, such as weak calcaneus following removal of the posterosuperior osseous prominence (7), persistent ache (8), and hypoesthesia in the area of the scar (9). The advantage of minimally invasive surgery is that it can be performed through a small incision, which heals very well. However, this technique does increase the operating time because radiology is used to position $\mathrm{K}$ wires in occasionally, and then, osteotomy occurs using a mechanical shaver or other intrument (5).

In most of the severe cases, after calcified lesion on Achilles tendon is removed, Achilles insertion point reconstruction is necessary. Anderson et al (10) suggest that if the point of dissected Achilles insertion was less than $50 \%$ of the total surface area, then the reconstruction cannot be implemented. If the point of dissected Achilles insertion was more 

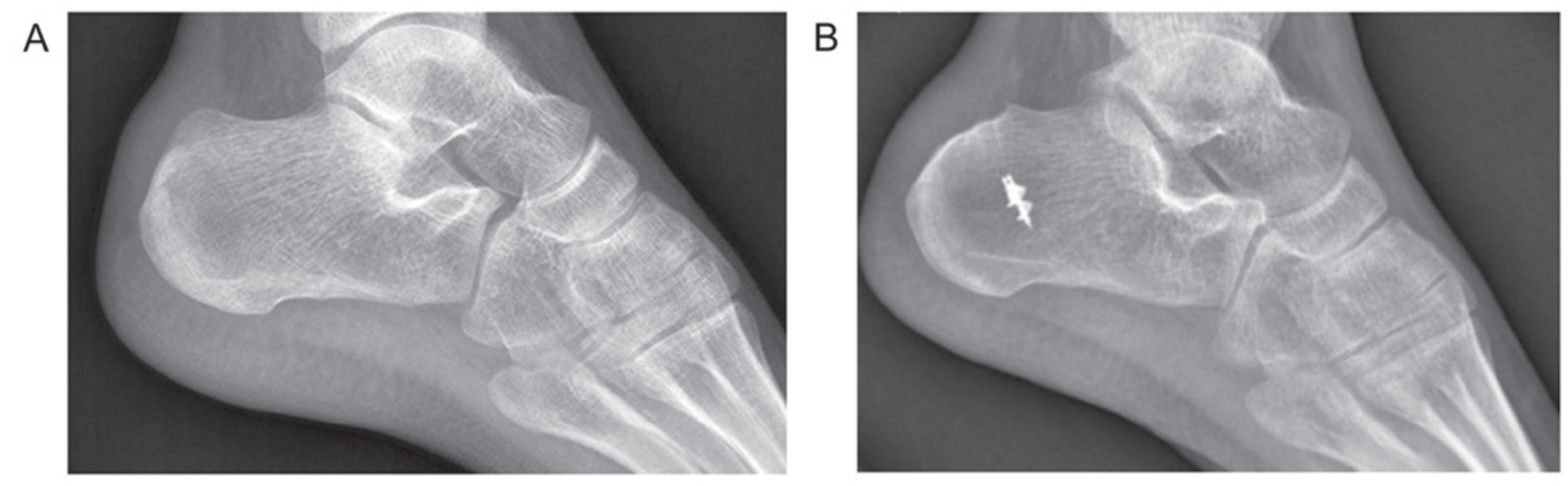

Figure 1. (A) Lateral film before operation shows posterosuperior calcaneal prominent obviously. (B) Lateral film after operation shows calcaneal prominence was removed completely.

than $50 \%$ of the total surface area, then the reconstruction can be implemented and fixed with two parenchyma anchors. In most of the studies, the anchors were implanted under direct vision, creating a larger wound. In 2013, Chiu et al suggested that acute Achilles tendon laceration can be repaired with modified percutaneous Bunnell suture treatment under endoscopy-assisted percutaneous repair (11).

We used this method in endoscopic Achilles tendon repair during the treatment of Haglund's syndrome. The suture anchors were implanted arthroscopically. The advantage of this method is that it avoids the occurrence of complications after traditional conservative treatment and open surgery. This method cannot only improve the suture effect and accuracy, but also retain the advantages of the operation of percutaneous tendon repair.

\section{Materials and methods}

Between September 2014 and July 2015, our technique was performed on 7 patients ( 3 female with 3 heels, 4 male with 4 heels), with an average age of 35.2 years. The average symptom time of all the patients was approximately 17.1 months (range, 12-24 months) before operation. Haglund's syndrome is mainly diagnosed by clinical symptoms, physical examination and lateral X-ray results as well as magnetic resonance imaging (MRI). The typical clinical symptoms of Haglund's syndrome include ankle Achilles tendon pain, redness, and swelling. In addition, all the patients with Haglund's syndrome exhibit an osseous prominence that directly affects their heel. All 7 patients were treated with lateral ankle X-ray and MRI examinations before operation. Standardized lateral ankle X-ray (Fig. 1A) of the weightbearing foot shows the prominence of posterosuperior calcaneus in the heel. The parallel pitch lines and Fowler and Philip angle (FPA) of each preoperative radiograph were measured. Posterosuperior calcaneal prominence of the patients had positive heel parallel pitch lines and FPA. The MRI results showed inflammation and swelling at the retrocalcaneal bursa and the soft tissue around the Achilles tendon insertion. Marrow edema was also observed in the posterosuperior calcaneal prominence of all 7 patients. MRI showed intratendinous calcifications in the 7 heels (Fig. 2).

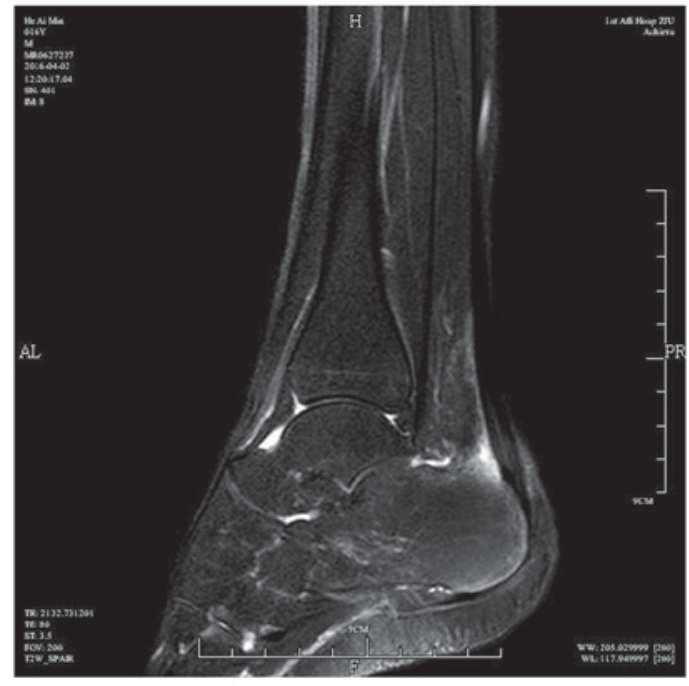

Figure 2. MRI of tendon calcification caused by Haglund's syndrome. MRI, magnetic resonance imaging.

None of the patients benefitted from conservative treatment, including avoidance of tight shoes, non-steroidal anti-inflammatory drugs, activity modification and physical therapy.

Surgical technique. Arthroscopic surgery occurred under lumbar or general anesthesia with the surgical patients in the prone position. A surgical tourniquet was applied to the thigh of the patient. The limb of the patient that was affected was elevated and set beyond the operating table. The first two portals were established as medial and lateral of the Achilles tendon and posterosuperior calcaneal prominence were at the same level. A $4 \mathrm{~mm}$ endoscopic arthroscope with $30^{\circ}$ field of view was placed as close as possible to the superior edge of the calcaneus, and also as far posterior as possible. If this portal was placed high, it would be difficult later during the procedure to reach the posteroinferior part of the calcaneus. A $4 \mathrm{~mm}$ shaver was inserted from the opposite portal. The posterior calcaneal fat pad was identified and removed. The bursectomy and posterior calcaneoplasty was performed as described by Jerosch (12). 


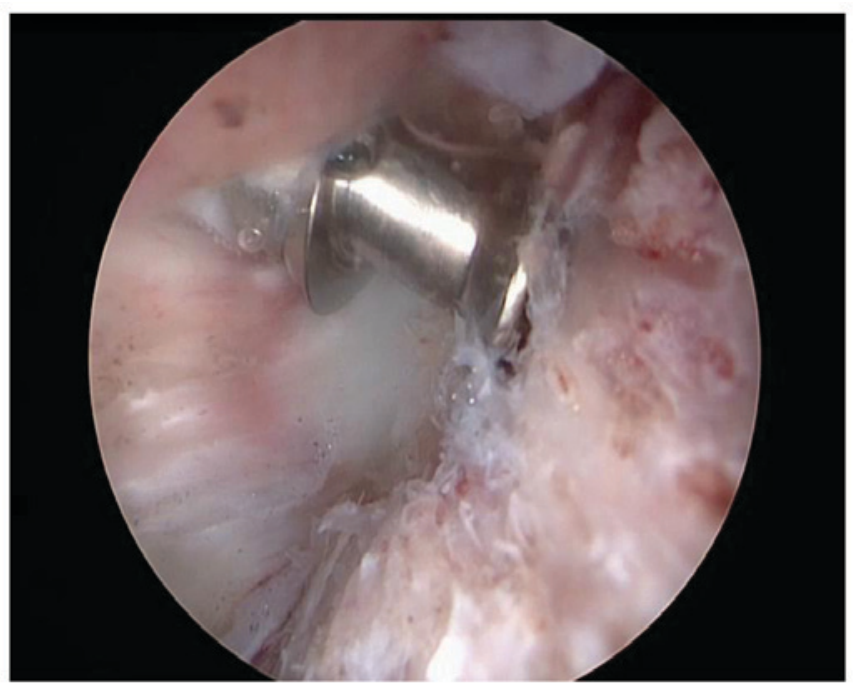

Figure 3. Suture anchors $(5.0 \mathrm{~mm})$ were implanted endoscopically in the Achilles tendon insertion site through the $4 \mathrm{~mm}$ incision.

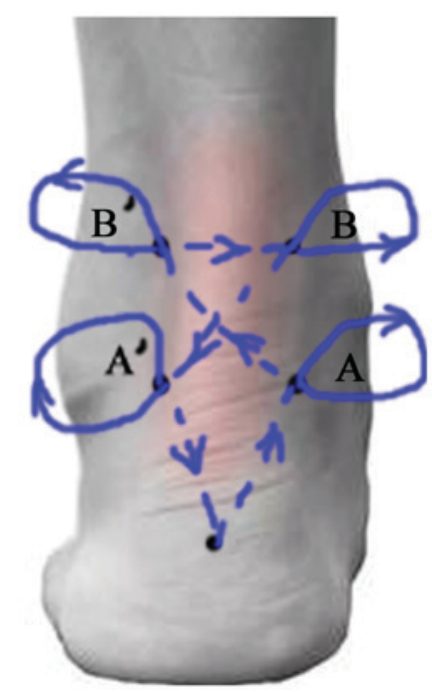

Figure 4 . The figure shows the 4 stab wounds and the incision site. The suture was passed from the incision wound to $\mathrm{A}$, then passed through the tendon and was drawn out. After that the needle was passed from A to B'. Then the needle was passed from portal $\mathrm{B}^{\prime}$ to $\mathrm{B}$, portal B to A', portal $\mathrm{A}^{\prime}$ back to incision wound and then knotted with another suture.

Partial tearing of the Achilles tendon insertion after the debridement was repaired with $5.0 \mathrm{~mm}$ suture anchors and modified Bunnell sutures immediately. A $4 \mathrm{~mm}$ incision was made at the posteromedial calcaneus. According to the dissection scope, 1 or 2 suture anchors were implanted in the Achilles tendon insertion site (Fig. 3). Four stab wounds were made above the insertion site, 2 at each side (Fig. 4). The suture was passed using a straight needle from the surgical incision wound to the lateral surgical wound (incision to A), through the heel tendon and drawn out. The operative needle was passed through the heel tendon and removed from the portal vein B (from portal vein A to B). Subsequently, the operative needle was passed from portal B' to B, portal B to A', portal A' back to the incision wound and then knotted with another suture. Of note, the whole needle had to be taken out and then pierced back at the 4 stab wounds.
Repetitious irrigation was used in the operation region. The incisions and stab wounds were sewn up and dressed with appropriate pressure.

Postoperative care. The leg was lifted on a pillow to reduce swelling at the first week after operation. Sutures were taken out 14 days later. Partial weightbearing activities wearing the Achilles walking boots were started 28 days postoperatively and full weight bearing activities 42 to 56 days postoperatively. The patient returned to normal activities and performed exercises 90 days postoperatively.

Statistical analysis. Parallel pitch lines of the patients and the American Orthopaedic Foot and Ankle Society (AOFAS) score were used to evaluate the clinical effect of patients at the last postoperative follow-up period. According to the patients' AOFAS score, the clinical curative effect was judged as: Poor curative (0-70 points), fair curative (70-79 points), good curative (80-89 points) and excellent curative effect (90-100 points) (13). The Wilcoxon two-sample test was used to detect the difference between the AOFAS score before and after operation. The results showed there was a significant difference between the AOFAS score before and after operation. $\mathrm{P}<0.05$ was considered to indicate a statistically significant difference. Statistical analysis was carried out using SPSS 20.0 software (IBM Inc., Armonk, NY, USA).

\section{Results}

All 7 patients underwent only one procedure and were followed up from 18 to 31 months (mean, 22 months). In all heels, the osseous prominence, retrocalcaneal bursitis and chondroid metaplasia of Achilles tendon insertion were observed under arthroscope (10). No Achilles tendon tear was reported. Negative parallel pitch lines and sufficient bony planning was shown in postoperative lateral position film (Fig. 1B). The average AOFAS score of the patients increased from $58.7 \pm 13.0$ points (before operation) to $87.8 \pm 12.7$ points (last follow-up period), and the difference was statistically significant.

Five of the 7 patients had excellent curative effect (90-100 points), 2 patients had good (80-89 points) and 0 patients had poor (0-70 points) or fair curative effect (70-79 points). None of the patients had postoperative nerve damage or postoperative permanent vascular injuries. In addition, there were no postoperative wound infections or postoperative Achilles tendon avulsions.

\section{Discussion}

Preoperative diagnosis of Haglund's syndrome holds significant value to the operation plan. The diagnosis of Haglund's syndrome was mainly based on the patients' clinical symptoms, physical examination, X-Ray, and MRI results. FPA has been observed to yield $86-100 \%$ false-negative results in previous studies (1,14-16). Lu et al (17) also suggested that the FPA and parallel pitch lines were not accurate indicators in reflecting the severity of symptomatic Haglund's syndrome. The surgeon should assess the surgery according to the patient's clinical symptoms. Radiographic measurements should be for reference only. MRI is commonly performed to assess the 
adjacent tendon for the need of intraoperative debridement concomitantly with the calcaneal osteoplasty. Insertional tendinosis is frequently associated with adjacent calcaneal marrow edema. This marrow edema was the most predictable sign of a symptomatic Achilles tendon in Haims et al MRI comparison of symptomatic and asymptomatic patients and has been shown to be a common associated finding in insertional tendinosis (18). Intraoperative endoscopic examination is useful when the ankle is passively dorsiflexed to observe the status of the patients' Achilles tendon along the calcaneus. If there is any pressure on the heel tendon with osseous prominence, posterior calcaneoplasty is considered feasible.

At present, large intratendinous calcifications correlated with heel chronic insertional Achilles tendinopathy limits the use of arthroscopic tenodesis (19). Arthroscopic planning of large prominences may lead to tendon insertional tearing if there is insufficient exposure (20). Jerosch (12) suggested that arthroscopic calcaneoplasty should be avoided in those types of ossific Achilles tendon insertions. Arthroscopic treatment may also be avoided in the intrinsic lesions of Achilles tendon.

Posterior midline approach and split of the Achilles tendon is preferred in those patients. Once posterosuperior calcaneal prominence is removed, the tendon is reattached with either transosseous sutures or bone anchors. Related research showed that bioabsorbable knotless screws with a spiked washer may be used in the operation of Achilles tendon reduction (20). Syed and Perera (5) suggested that a small amount of tendon elevation does not require reattachment. However, if this is significant, then suture anchors can be passed percutaneously through the tendon and the skin opened superficially to allow these to be tied off. Alternatively, these can be tied off internally by pulling the sutures through into the retrocalcaneal space, out of the instrument portal and tied off under direct vision with a knot pusher. Ma and Griffith suggested (21) the development of acute closed ruptured Achilles tendon percutaneous repair firstly, followed by some treatment modifications, including heel repair of Achilles tendon with plantaris tendon augmentation (22-26). Previous findings have shown that acute Achilles tendon rupture repair with endoscopy can repair the rears of Achilles tendon and finish the strengthening through the modification of Bunnell sutures (11). This method can effectively avoid the incidence of complications, ensure the good repairing effect of the patient's skin and tendon and improve the accuracy of suture after surgery. Wiegerinck et al (27) report that no minimal invasive surgical studies offer a clear method to reinsert the Achilles tendon with bone anchors or perform augmentation (plantaris tendon or FHL). In our practice, when the sutures were tied with a knot guide under endoscopy, the ankle was held to perform plantar flexion within a certain range of motion under the doctor's guidance. The gap between tendon and calcaneus was checked through the endoscope in real time. Less ankle plantar flexion was performed and tied relatively when the clearance distance was small after detection. If the clearance distance was large enough, it was tied with stronger ankle dorsiflexion. Thus, surgeons can reach a solid and suitable fixation of the torn tendon end.

Our study is a supplement of endoscopic repair and strengthening of the Achilles tendon. It expands the indication of arthroscopic therapy of Haglund's syndrome and is a reliable technique.

Study limitations include lack of a control group (only 3 cases were compared with self control). As the number of subjects included was less, the postoperative follow-up time was relatively short. Therefore, more subjects are required in prospective research to verify our results. Theoretically, our technique is also suitable for Achilles tendon augmentation or the repair of full rupture of Achilles tendon after intratendinous debridement.

\section{Competing interests}

The authors declare that they have no competing interests.

\section{References}

1. Chang C-D and Wu JS: MR Imaging findings in heel pain. Magn Reson Imaging Clin N Am 25: 79-93, 2017.

2. Wiegerinck JI, Kok AC and van Dijk CN: Surgical treatment of chronic retrocalcaneal bursitis. Arthroscopy 28: 283-293, 2012.

3. Wu Z, Hua Y, Li Y and Chen S: Endoscopic treatment of Haglund's syndrome with a three portal technique. Int Orthop 36: 1623-1627, 2012.

4. Jerosch J and Nasef NM: Endoscopic calcaneoplasty-rationale, surgical technique, and early results: A preliminary report. Knee Surg Sports Traumatol Arthrosc 11: 190-195, 2003.

5. Syed TA and Perera A: A proposed staging classification for minimally invasive management of Haglund's syndrome with percutaneous and endoscopic surgery. Foot Ankle Clin 21: 641-664, 2016.

6. Frey C: Surgical advancements: Arthroscopic alternatives to open procedures: Great toe, subtalar joint, Haglund's deformity, and tendoscopy. Foot Ankle Clin 14: 313-339, 2009.

7. Perlman MD: Enlargement of the entire posterior aspect of the calcaneus: Treatment with the Keck and Kelly calcaneal osteotomy. J Foot Surg 31: 424-433, 1992.

8. Nesse E and Finsen V: Poor results after resection for Haglund's heel. Analysis of 35 heels in 23 patients after 3 years. Acta Orthop Scand 65: 107-109, 1994

9. Pauker M, Katz K and Yosipovitch Z: Calcaneal ostectomy for Haglund disease. J Foot Surg 31: 588-589, 1992.

10. Anderson JA, Suero E, O'Loughlin PF and Kennedy JG: Surgery for retrocalcaneal bursitis: A tendon-splitting versus a lateral approach. Clin Orthop Relat Res 466: 1678-1682, 2008.

11. Chiu CH, Yeh WL, Tsai MC, Chang SS, Hsu KY and Chan YS: Endoscopy-assisted percutaneous repair of acute Achilles tendon tears. Foot Ankle Int 34: 1168-1176, 2013.

12. Jerosch J: Endoscopic calcaneoplasty. Foot Ankle Clin 20: $149-165,2015$.

13. Jardé O, Quenot P, Trinquier-Lautard JL, Tran-Van F and Vives P: Haglund disease treated by simple resection of calcaneus tuberosity. An angular and therapeutic study. A propos of 74 cases with 2 years follow-up. Rev Chir Orthop Repar Appar Mot 83: 566-573, 1997 (In France).

14. Heneghan MA and Pavlov H: The Haglund painful heel syndrome. Experimental investigation of cause and therapeutic implications. Clin Orthop Relat Res 187: 228-234, 1984.

15. Ruch JA: Haglund's disease. J Am Podiatry Assoc 64: 1000-1003, 1974.

16. Fuglsang F and Torup D: Bursitis retrocalcanearis. Acta Orthop Scand 30: 315-323, 1961.

17. Lu CC, Cheng YM, Fu YC, Tien YC, Chen SK and Huang PJ: Angle analysis of Haglund syndrome and its relationship with osseous variations and Achilles tendon calcification. Foot Ankle Int 28: 181-185, 2007.

18. Haims AH, Schweitzer ME, Patel RS, Hecht P and Wapner KL: MR imaging of the Achilles tendon: Overlap of findings in symptomatic and asymptomatic individuals. Skeletal Radiol 29: 640-645, 2000.

19. Leitze Z, Sella EJ and Aversa JM: Endoscopic decompression of the retrocalcaneal space. J Bone Joint Surg Am 85-A: 1488-1496, 2003. 
20. Maquirriain J: Endoscopic Achilles tenodesis: A surgical alternative for chronic insertional tendinopathy. Knee Surg Sports Traumatol Arthrosc 15: 940-943, 2007.

21. Ma GW and Griffith TG: Percutaneous repair of closed ruptured achilles tendon: A new technique. Clin Orthop Relat Res 128 247-255, 1977.

22. Assal M, Jung M, Stern R, Rippstein P, Delmi M and Hoffmeyer P: Limited open repair of Achilles tendon ruptures: A technique with a new instrument and findings of a prospective multicenter study. J Bone Joint Surg Am 84-A: 161-170, 2002.

23. Cretnik A, Kosanović M and Smrkolj V: Percutaneous suturing of the ruptured Achilles tendon under local anesthesia. J Foot Ankle Surg 43: 72-81, 2004.

24. Kakiuchi M: A combined open and percutaneous technique for repair of tendo Achillis. Comparison with open repair. J Bone Joint Surg Br 77: 60-63, 1995.
25. Lui TH: Endoscopic-assisted Achilles tendon repair with plantaris tendon augmentation. Arthroscopy 23: 556.e1-556.e5, 2007.

26. Majewski M, Rohrbach M, Czaja S and Ochsner P: Avoiding sural nerve injuries during percutaneous Achilles tendon repair. Am J Sports Med 34: 793-798, 2006.

27. Wiegerinck JI, Zwiers R, van Sterkenburg MN, Maas MM and van Dijk CN: The appearance of the pre-Achilles fat pad after endoscopic calcaneoplasty. Knee Surg Sports Traumatol Arthrosc 23: 2400-2405, 2015.

This work is licensed under a Creative Commons Attribution-NonCommercial-NoDerivatives 4.0 International (CC BY-NC-ND 4.0) License. 